\title{
Characterization of a THz SLM and its application for improved high resolution $\mathrm{THz}$ imaging
}

\author{
J. Hieronymus ${ }^{1}$, S. Augustin ${ }^{1,2}$, and H.-W. Hübers ${ }^{1,3}$ \\ ${ }^{1}$ German Aerospace Center (DLR), Rutherfordstr. 2, 12489 Berlin, Germany \\ ${ }^{2}$ Federal Institute for Materials Research and Testing (BAM), Unter den Eichen 8712205 Berlin, Germany \\ ${ }^{3}$ Humboldt-Universität zu Berlin, Department of Physics, Newtonstr. 5, 12489 Berlin, Germany
}

\begin{abstract}
Spatial light modulators (SLM) can be used for many applications, including imaging and beam steering. With the emergence of THz SLMs being able to control thousands of pixels, the aforementioned applications become feasible for the THz region. The degree of control that can be exerted with a SLM and in turn its usefulness directly depends on the light pattern it creates. The resulting light patterns can be quite different from the intended light patterns, due to systematic and random non-uniformities, especially for large $\mathrm{THz}$ wavelengths. Here we present results on the performance of a Germanium based THz SLM. These results enable the efficient application of Compressed Sensing techniques in THz SLM imaging settings.
\end{abstract}

\section{INTRODUCTION}

$\mathrm{T}$ $\mathrm{Hz}$ imaging nowadays can be realized with novel setups that are capable of acquiring images without the need for mechanical scanning. A core component of such a design is a spatial light modulator (SLM). In general, SLMs are used to manipulate incoming radiation spatially. A SLM typically consists of an array of rectangular filter elements, which can be commanded to be transparent or non-transparent (non-reflective or reflective) for the employed radiation. For the $\mathrm{THz}$ region there are different approaches of constructing such SLMs. The type of THz SLM we analyze in this paper is based on a digital micromirror devive (DMD) for visible wavelengths in combination with a Germanium disc that acts as optical switch. The Germanium is illuminated with spatially structured patterns via the DMD. The semiconductor translates the structured patterns from the visual part of the spectrum to the $\mathrm{THz}$ region. A notable advantage of this type of THz SLM is the large number of pixels readily available.

\section{EXPERIMENTAL SETUP AND RESULTS}

The THz SLM characterized in this paper is used for nonmechanical $\mathrm{THz}$ imaging in a single-pixel setup, which is shown in Fig. 1. The incoming $\mathrm{THz}$ beam from a $0.35 \mathrm{THz}$ multiplier source illuminates the Germanium disc. In addition the light from a halogen tungsten lamp is imaged onto a DMD which reflects the light onto the Germanium disc. The computer-controlled DMD generates different spatial light patterns (so called masks). Areas on the Germanium disc that are illuminated act as reflectors/attenuators for $\mathrm{THz}$ radiation, while areas that are not illuminated transmit the $\mathrm{THz}$ radiation. The transmitted radiation is detected by a $0.35 \mathrm{THz}$ heterodyne receiver. For each mask a measurement is taken. In a following step, the object $(\mathrm{x})$ is reconstructed based on the knowledge of the applied masks $(\Phi)$ and the corresponding measurements (y) using Compressed Sensing methods. Even though, the masks that were commanded are perfectly known, the resulting $\mathrm{THz}$ patterns may differ in several ways. The better the knowledge of these physically applied $\mathrm{THz}$ filtermasks, the better the reconstruction results, in terms of image quality and resolution.

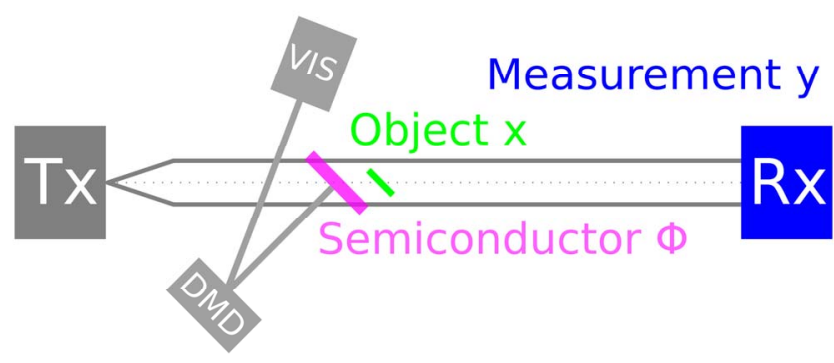

Fig. 1. Scheme of the experimental setup. Dark gray: THz radiation. Light gray: visible light. Purple: Germanium Disc. Green: Object to be imaged. Blue $\mathrm{THz}$ receiver.

In order to investigate the $\mathrm{THz}$ masks, they have been characterized by scanning a Golay cell detector mounted onto mechanical $x-y$-scanner. In Fig. 2a) a raster scanned $\mathrm{THz}$ mask is shown along with its corresponding commanded mask (Fig. 2b)). In the outer area (black) the signal was too low to be detected.

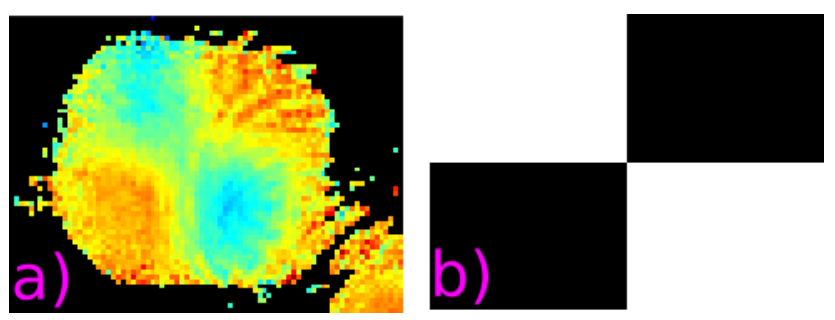

Fig. 2. a) Raster scan of a $2 \times 2$ chessboard THz mask ; b) commanded mask, white being illuminated areas, resulting in higher attenuation and therefore lower $\mathrm{THz}$ signal

In Fig. 3 an example of a reconstructed image is shown. 

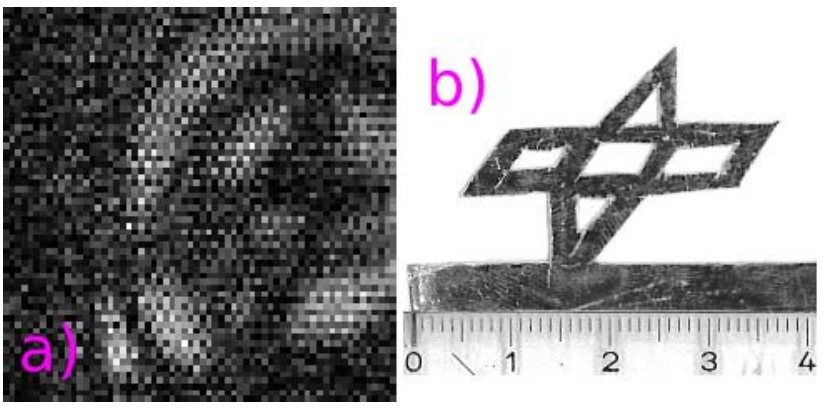

Fig. 3. a) THz image with $64 \times 64$ pixels of size approx. $0.7 \mathrm{~mm}$ each, without optimizing the image reconstruction using the characterization of the SLM.

b) Visual image of the object (DLR logo, made from aluminum).

The image in Fig. 3 is reconstructed from a set of random masks. The corresponding measured signals (y), depicted in blue in Fig. 4, seem to be random, as expected. Note however, that their standard deviation is about a factor two larger than the standard deviation of the reference black masks (black) and reference white masks (cyan). This is a clear indicator, that there is information in the measured signal. This information can be extracted, together with the knowledge of the commanded mask and the knowledge of the behavior of the THz SLM using either standard methods like inverting a linear system or more advanced tools from the Compressed Sensing domain.

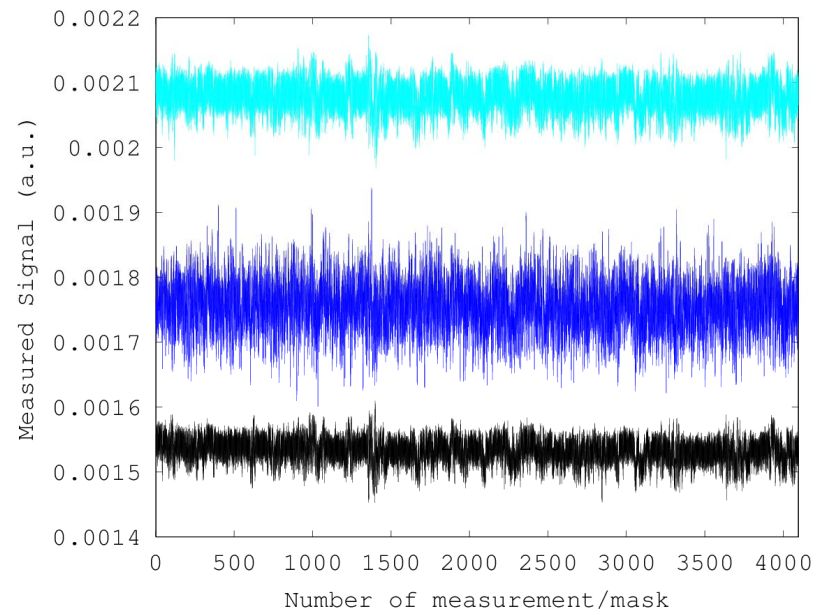

Fig. 4. Measurements taken during a replay of a set of random masks (blue). Cyan and black indicate reference signal levels for fully white and fully black masks respectively.

\section{SUMMARY}

We have demonstrated the characterization of a THz SLM used in a non-mechanical single-pixel $\mathrm{THz}$ imaging application. The distinct knowledge of certain properties of the $\mathrm{THz}$ SLM provides the necessary tools to improve the imaging capability of SLM based $\mathrm{THz}$ imager.

\section{ACKNOWLEDGEMENTS}

J. Hieronymus gratefully acknowledges support by the Helmholtz Research School on Security Technologies (HRSST).

\section{REFERENCES}

[1]. S. Augustin, J. Hieronymus, P. Jung and H.-W. Hübers, "Compressed Sensing in a Fully Non-Mechanical $350 \mathrm{GHz}$ Imaging Setting," Journal of Infrared, Millimeter, and Terahertz Waves, vol. 36, pp. 496-512, January, 2015. 